\title{
Nowhere differentiable functions of analytic type on products of finitely connected planar domains
}

\author{
Vlasios Mastrantonis $^{1,2}$. Christoforos Panagiotis ${ }^{3}$
}

Received: 3 June 2017 / Accepted: 25 October 2017 / Published online: 8 November 2017

(C) The Author(s) 2017. This article is an open access publication

\begin{abstract}
Using Laurent decomposition and Mergelyan's theorem combined with Baire's category theorem, we prove generic nowhere differentiability on the distinguished boundary of functions of analytic type on products of planar domains bounded by finitely many disjoint Jordan curves. The parametrization of the boundaries are those induced by the natural Riemann maps.
\end{abstract}

Keywords Nowhere differentiable functions - Baire's theorem - Riemann mapping · Rational approximation · Several complex variables $\cdot$ Laurent decomposition

Mathematics Subject Classification Primary 26A27; Secondary 30H50

\section{Introduction}

In the nineteenth century, it was widely believed that every continuous function defined on $\mathbb{R}$ has derivative at a significant set of points. Weierstrass was the first one to

\section{Communicated by A. Constantin.}

Supported by the European Research Council (ERC) under the European Union's Horizon 2020 research and innovation programme (Grant Agreement No. 639046). Research was conducted at the University of Athens, Department of Mathematics.

$凶$ Vlasios Mastrantonis

vm367@cam.ac.uk

Christoforos Panagiotis

C.Panagiotis@warwick.ac.uk

1 Department of Mathematics, University of Athens, Panepistimioupolis, 157 84, Athens, Greece

2 Present Address: DPMMS, University of Cambridge, Centre for Mathematical Sciences, Wilberforce Road, Cambridge CB3 OWB, UK

3 Mathematics Institute, University of Warwick, Coventry CV4 7AL, UK 
disprove this claim by providing an explicit example of a function $u: \mathbb{R} \rightarrow \mathbb{R}$ which is continuous, $2 \pi$-periodic, but not differentiable at any real number. Since then the existence of such functions attracted the interest of many mathematicians.

In fact, as Banach and Mazurkiewicz independently proved, in [2] and [9] respectively, that a topologically generic continuous function on a compact interval $J$ of $\mathbb{R}$ is nowhere differentiable. In [3], an analogue of this theorem has been proved for functions in the disc algebra $A(D)$, i.e. continuous functions defined on the closed unit disc $\bar{D}$, which are also holomorphic on $D$. In particular, it is proved that generically for every function $f \in A(D)$ both functions $\operatorname{Re} f \Upsilon_{\mathbb{T}}$ and $\operatorname{Im} f \Upsilon_{\mathbb{T}}$ are nowhere differentiable. The result is even extended for functions $f: \bar{D}^{I} \rightarrow \mathbb{C}$ that belong to the algebra $A\left(D^{I}\right)$, namely $f$ is continuous on $\bar{D}^{I}$, which is endowed with the cartesian topology, and separately holomorphic on $D^{I}$.

In Sect. 4 , we prove a similar result in the case of a domain $\Omega$ bounded by a finite number of disjoint Jordan curves. If $\Omega_{0}, \Omega_{1}, \ldots \Omega_{n}$ are the complements of the connected components of $(\mathbb{C} \cup\{\infty\}) \backslash \Omega$, then the Riemann mappings $\phi_{i}: \bar{D} \rightarrow \overline{\Omega_{i}}$, $i=1, \ldots, n$ yield a parametrization of $\partial \Omega_{i}, i=0,1, \ldots, n$, with parameter $\theta \in \mathbb{R}$. We will prove that generically for every $f \in A(\Omega)$ the functions $\operatorname{Re}\left(f \circ \phi_{i}\right) \Upsilon_{\mathbb{T}}$ and $\operatorname{Im}\left(f \circ \phi_{i}\right)\left\lceil_{\mathbb{T}}\right.$ are nowhere differentiable with respect to $\theta \in \mathbb{R}$. In order to do so, we combine the Laurent decomposition of every function $f \in A(\Omega)$ with the results of the previous sections which deal with the case of the disc or more generally with the case of Jordan domains.

In Sect. 5, we generalize the above result in the context of several complex variables. Specifically, if we consider sets $\Omega$ which are the finite or countably infinite cartesian products of domains in $\mathbb{C}$ as in Sect. 4 , then a parametrization of the distinguished boundary of $\Omega$ arises from the Riemann mappings of the factors $\Omega_{i}$ of the product $\Omega$. We prove that, generically, for every $f \in A(\Omega)$ neither the real part $\operatorname{Re} f$, nor the imaginary part $\operatorname{Im} f$ have directional derivatives at any point of the distinguished boundary of $\Omega=\prod_{i \in I} \Omega_{i}$ with respect to the above parametrization, for any direction $v \in \ell^{\infty} \backslash\{0\}$. We first treat the case of finite products that is when the set $I$ is finite. In the case of infinitely many variables, we use the result for the case of finitely many variables and the fact that every $f \in A(\Omega)$ can be uniformly approximated by functions depending on a finite set of variables $([4,8])$.

\section{The open unit disk}

We denote the open unit disk in $\mathbb{C}$ by $D$ and $\mathbb{T}=\partial D$. Let $\Omega$ be a domain in $\mathbb{C}$. Although the only domains we will deal with in this sections are $D$ and $\hat{\mathbb{C}} \backslash \bar{D}$ we will give a general definition which will be useful later.

Definition 2.1 Let $\Omega$ be a domain in $\mathbb{C}$. A function $f: \bar{\Omega} \rightarrow \mathbb{C}$ belongs to $A(\Omega)$ if it is continuous on $\bar{\Omega}$ and holomorphic on $\Omega$. We endow $A(\Omega)$ with the topology of uniform convergence on compact subsets of $\bar{\Omega}$, where the closure of $\Omega$ is taken in $\mathbb{C}$.

We note that a function defined on $\mathbb{T}$ can equivalently be thought as a $2 \pi$-periodic function defined on $\mathbb{R}$. Thus, by abuse of notation we write $u(y)$ instead of $u\left(e^{i y}\right)$ for $y \in \mathbb{R}$. 
Definition 2.2 A function $f \in C(\mathbb{T})$ belongs to $Z$ if for every $\theta \in \mathbb{R}$

$$
\limsup _{y \rightarrow \theta^{+}}\left|\frac{\operatorname{Re} f(y)-\operatorname{Re} f(\theta)}{y-\theta}\right|=+\infty
$$

and

$$
\limsup _{y \rightarrow \theta^{+}}\left|\frac{\operatorname{Im} f(y)-\operatorname{Im} f(\theta)}{y-\theta}\right|=+\infty .
$$

In [3], it has been proven that the set of functions $f \in A(D)$ such that $f\lceil\mathbb{T}$ belongs to $Z$, which we denote by $Z(D)$, is a dense and $G_{\delta}$ subset of the disk algebra $A(D)$.

It will be useful to restrict ourselves to functions $f \in A(D)$, such that $f(0)=0$. We denote the space of such functions by $A_{0}(D)$ and we endow it with the subspace topology. Using the above result we are going to prove that the functions $f \in A_{0}(D)$ such that $f\left\lceil_{\mathbb{T}} \in Z\right.$ is dense and $G_{\delta}$ in $A_{0}(D)$. Denote this set by $Z_{0}(D)$.

Proposition 2.3 The set of functions $f \in A_{0}(D)$ such that $f \uparrow \mathbb{T}$ belongs to $Z$ is a dense and $G_{\delta}$ subset of $A_{0}(D)$.

Proof Observe that $Z_{0}(D)=Z(D) \cap A_{0}(D)$. Consider the mapping

$$
\begin{aligned}
L: A_{0}(D) & \longrightarrow A(D) \\
f & \longmapsto f .
\end{aligned}
$$

$L$ is obviously continuous and it is easy to see that $L^{-1}(Z(D))=Z_{0}(D)$ is $G_{\delta}$. So it suffices to show that $Z_{0}$ is dense in $A_{0}(D)$.

Let $\epsilon>0$ and $f \in A_{0}(D) \subset A(D)$. Since $Z(D)$ is dense in $A(D)$ there exists a $g \in Z(D)$ such that

$$
\|f-g\|_{\infty}<\frac{\epsilon}{2}
$$

More specifically

$$
|f(0)-g(0)|<\frac{\epsilon}{2} .
$$

It is now obvious that $\tilde{g}(z)=g(z)-g(0) \in A_{0}(D)$ and $\tilde{g} \in Z_{0}(D)$. Using the triangular inequality we deduce that

$$
\|f-\tilde{g}\|_{\infty}<\epsilon
$$

which completes the proof.

We will now prove similar results for a subspace of $A(\hat{\mathbb{C}} \backslash \bar{D})$ denoted by $A_{0}(\hat{\mathbb{C}} \backslash \bar{D})$. In general, if the domain $\Omega$ is the complement of a compact set, then a function $f$ belongs to $A_{0}(\Omega)$ if $f \in A(\Omega)$ and

$$
\lim _{z \rightarrow \infty} f(z)=0
$$


Proposition 2.4 The set of functions $f \in A_{0}(\hat{\mathbb{C}} \backslash \bar{D})$ such that $f \uparrow_{\mathbb{T}} \in Z$ is a dense and $G_{\delta}$ subset of $A_{0}(\hat{\mathbb{C}} \backslash \bar{D})$.

Proof Consider the mappings

$$
\begin{gathered}
\phi: \bar{D} \longrightarrow \hat{\mathbb{C}} \backslash D \\
z \longmapsto \frac{1}{z}
\end{gathered}
$$

and

$$
\begin{aligned}
l: A_{0}(\hat{\mathbb{C}} \backslash \bar{D}) & \longrightarrow A_{0}(D) \\
f & \longmapsto f \circ \phi .
\end{aligned}
$$

We can easily see that $l$ is continuous, onto $A_{0}(D)$ and

$$
\|l(f)-l(g)\|_{\infty}=\|f-g\|_{\infty} .
$$

Hence, $l$ is an isometric isomorphism, which implies that the set $Z_{0}(\hat{\mathbb{C}} \backslash \bar{D}):=$ $l^{-1}\left(Z_{0}(D)\right)$ is dense and $G_{\delta}$ in $A_{0}(\hat{\mathbb{C}} \backslash \bar{D})$.

We will now prove that $Z_{0}(\hat{\mathbb{C}} \backslash \bar{D})$ coincides with the set of functions $f \in A_{0}(\hat{\mathbb{C}} \backslash \bar{D})$ such that $f \uparrow_{\mathbb{T}} \in Z$. This can be derived from the following equalities:

$$
\begin{aligned}
& \left|\frac{u(y)-u(\theta)}{y-\theta}\right|=\left|\frac{(u \circ \phi)(-y)-(u \circ \phi)(-\theta)}{(-y)-(-\theta)}\right|, \\
& \left|\frac{v(y)-v(\theta)}{y-\theta}\right|=\left|\frac{(v \circ \phi)(-y)-(v \circ \phi)(-\theta)}{(-y)-(-\theta)}\right|,
\end{aligned}
$$

where $u, v$ are the real and imaginary part of a function $f \in A_{0}(D)$ respectively and $\theta, y \in \mathbb{R}$ are such that $y \neq \theta$.

\section{Jordan domains}

In this section we replace the unit circle $\mathbb{T}$ by a Jordan curve $J$ and we prove similar results for functions defined on the Jordan domain $\Omega$ or on $\hat{\mathbb{C}} \backslash \bar{\Omega}$. The Jordan curve theorem reassures that the complement of a Jordan curve in $\mathbb{C}$ has exactly two connected components, one bounded and one unbounded. We define a Jordan domain to be the bounded connected component of the complement of a Jordan curve on $\mathbb{C}$. For such domains $\Omega$, every Riemann mapping $\phi: D \rightarrow \Omega$ has a continuous and injective extension over the closed the unit disk $\bar{D}$ ([7]). We claim that generically for every function $f \in A(\Omega)$, the function $(f \circ \phi)\left\lceil_{\mathbb{T}}\right.$ belongs to $Z$. In what follows, we prove the aforementioned assertions.

Theorem 3.1 Let $\Omega$ be a Jordan domain and $\phi: \bar{D} \rightarrow \bar{\Omega}$ a Riemann mapping. The set of functions $f \in A(\Omega)$ such that $(f \circ \phi) \uparrow_{\mathbb{T}}$ belongs to $Z$ is a dense and $G_{\delta}$ subset of $A(\Omega)$. 
Proof Let

$$
\begin{aligned}
L: A(\Omega) & \longrightarrow A(D) \\
f & \longmapsto f \circ \phi .
\end{aligned}
$$

It is easy to verify that $L$ is an isometric isomorphism, which implies that the set $L^{-1}(Z(D))$ is a dense and $G_{\delta}$ subset of $A(\Omega)$.

Furthermore $L^{-1}(Z(D))$ coincides with the set of functions $f \in A(\Omega)$ such that $(f \circ \phi) \Upsilon_{\mathbb{T}}$ belongs to $Z$, because, for any $f \in A(\Omega)$ we have that: $(f \circ \phi) \Upsilon_{\mathbb{T}} \in Z \Leftrightarrow$ $L(f) \uparrow_{\mathbb{T}} \in Z \Leftrightarrow L(f) \in Z(D) \Leftrightarrow f \in L^{-1}(Z(D))$.

Theorem 3.2 Let $J$ be a Jordan curve and $\Omega$ the unbounded connected component of $\mathbb{C} \backslash J^{*}$. Let also $\phi: \bar{D} \rightarrow \overline{\Omega \cup\{\infty\}}$ be a Riemann mapping. The set of functions $f \in A(\Omega \cup\{\infty\})$ such that $(f \circ \phi) \Upsilon_{\mathbb{T}}$ belongs to $Z$ is a dense and $G_{\delta}$ subset of $A_{0}(\Omega \cup\{\infty\})$.

Proof Consider the mapping

$$
\begin{aligned}
\mathcal{L}: A_{0}(\Omega \cup\{\infty\}) & \longrightarrow A_{0}(D) \\
f & \longmapsto f \circ \phi .
\end{aligned}
$$

Similarly to Proposition 2.4 the desired set is a dense and $G_{\delta}$ subset of $A_{0}(\Omega$ $\cup\{\infty\})$.

Remark 3.3 In Theorems 3.1 and 3.2 every Riemann mapping $\Phi: D \rightarrow \Omega$ can be obtained from the composition of a fixed Riemann mapping $\phi$ of $\Omega$ with a Möbius transformation mapping $D$ onto itself. Therefore, one can easily see that if the functions $\operatorname{Re}(f \circ \phi)\left\lceil_{\mathbb{T}}\right.$ and $\operatorname{Im}(f \circ \phi) \Upsilon_{\mathbb{T}}$ are nowhere differentiable with respect to $\theta \in \mathbb{R}$, then the same holds true for the functions $\operatorname{Re}(f \circ \Phi)\left\lceil_{\mathbb{T}}\right.$ and $\operatorname{Im}(f \circ \Phi)\left\lceil_{\mathbb{T}}\right.$.

The statement of Remark 3.3 does not hold in general if we replace $\Phi$ by an arbitrary parametrization. In what follows we prove that if a function $f_{0} \in A(D)$ is nowhere differentiable with respect to the natural parametrization and $\operatorname{Re} f_{0} \Upsilon_{\mathbb{T}}, \operatorname{Im} f_{0} \Upsilon_{\mathbb{T}}$ are $a$ Hölder continuous, then there is a parametrization $s$ of $\mathbb{T}$ such that $f_{0} \Upsilon_{\mathbb{T}}$ is differentiable at some point with respect to $s$.

Suppose that $f_{0}$ is a function as above. Let $s(t)=t^{b}$ for some $b>1 / a$. It is easy to see that $\operatorname{Re}\left(f_{0} \circ s\right)\left\lceil_{\mathbb{T}}\right.$ and $\operatorname{Im}\left(f_{0} \circ s\right) \uparrow_{\mathbb{T}}$ are differentiable at 0 with respect to $s$ and their derivatives are equal to 0 . Indeed, this follows from the fact that

$$
\frac{\left|u_{0}(s(t))-u_{0}(s(0))\right|}{|t|} \leq c \frac{|t|^{a b}}{|t|}
$$

for some constant $c>0$, where $u_{0}=\operatorname{Re} f_{0}$. The last expression converges to 0 as $t \rightarrow 0$, which implies that the derivative of $\left(u_{0} \circ s\right) \Upsilon_{\mathbb{T}}$ at 0 is equal to 0 . Similarly, the derivative of $\operatorname{Im}\left(f_{0} \circ s\right) \uparrow_{\mathbb{T}}$ at 0 is 0 . 
To give an example of such a function $f_{0}$ consider the Weierstrass function $u_{0}$ : $\mathbb{R} \rightarrow \mathbb{R}$ defined by

$$
u_{0}(x)=\sum_{n=0}^{\infty} 2^{-a n} \cos \left(2^{n} x\right)
$$

for $0<a<1$. Since $u_{0}$ is $2 \pi$-periodic, we can regard $u_{0}$ as a function defined on $\mathbb{T}$. Note that $u_{0}=\operatorname{Re}\left(f_{0}\right)$ where $f_{0}(z)=\sum_{n=0}^{\infty} 2^{-a n} z^{2^{n}}$. The latter function belongs to $A(D)$ as the M-test shows. A classic result of G. H. Hardy [6] states that both $u_{0}$ and its harmonic conjugate $\tilde{u}_{0}$ are nowhere differentiable and $a$-Hölder continuous.

On the other hand, a nowhere differentiable function with respect to a parametrization $s_{1}$ of a Jordan curve $J$ is also nowhere differentiable with respect to another parametrization $s_{2}$ whenever they satisfy $s_{2}=s_{1} \circ r$ for a differentiable function $r: \mathbb{T} \rightarrow \mathbb{T}$ with $r^{\prime}(t) \neq 0$ for every $t$. Indeed, consider a function $f \in A(\Omega)$ such that $\left(f \circ s_{1}\right)\left\lceil_{\mathbb{T}} \in Z\right.$. Then we have that

$$
\frac{f\left(s_{2}(t)\right)-f\left(s_{2}\left(t_{0}\right)\right)}{t-t_{0}}=\frac{f\left(s_{1}(r(t))\right)-f\left(s_{1}\left(r\left(t_{0}\right)\right)\right)}{r(t)-r\left(t_{0}\right)} \frac{r(t)-r\left(t_{0}\right)}{t-t_{0}}
$$

which implies that $\left.\left(f \circ s_{2}\right)\right|_{\mathbb{T}} \in Z$. Hence, a nowhere differentiable function with respect to some parametrization is nowhere differentiable with respect to every other "nice" reparametrization.

\section{Domains bounded by a finite number of disjoint Jordan curves}

Now we consider a more general setting, where $\Omega$ is a bounded domain whose boundary consists of a finite number of disjoint Jordan curves. If $V_{0}, \ldots, V_{n-1}$ are the connected components of $\hat{\mathbb{C}} \backslash \Omega, \infty \in V_{0}$ and $\Omega_{0}=\hat{\mathbb{C}} \backslash V_{0}, \ldots, \Omega_{n-1}=\hat{\mathbb{C}} \backslash V_{n-1}$, then there exist Riemann mappings $\phi_{i}: \bar{D} \rightarrow \overline{\Omega_{i}}$. We are interested in functions $f \in A(\Omega)$, such that $\left(f \circ \phi_{i}\right) \Upsilon_{\mathbb{T}}$ belongs to $Z$, for all $i=0, \ldots, n-1$.

The main result of this section is that the aforementioned class of functions is $G_{\delta}$ dense in $A(\Omega)$.

Theorem 4.1 The class of functions $f \in A(\Omega)$ such that for every $i \in\{0,1, \ldots, n-$ $1\}$, the functions $f \circ \phi_{i} \in Z$ is dense and $G_{\delta}$ in $A(\Omega)$.

We will prove the theorem using the results of the previous sections, the Laurent decomposition and Baire category theorem.

At first let us give some necessary definitions. For every $k \in \mathbb{N}$ and $i \in$ $\{0,1, \ldots, n-1\}$ consider the sets

$$
\begin{gathered}
D_{k}=\left\{u \in C_{\mathbb{R}}(T) \text { : for every } \theta \in \mathbb{R} \text { there exists } y \in\left(\theta, \theta+\frac{1}{k}\right)\right. \\
\text { such that }|u(y)-u(\theta)|>k|y-\theta|\}
\end{gathered}
$$


and

$$
E_{k}^{(i)}=\left\{f \in A(\Omega): \operatorname{Re}\left(f \circ \phi_{i}\right) \uparrow_{\mathbb{T}} \in D_{k}\right\}
$$

Lemma 4.2 For every $k \in \mathbb{N}$ and $i \in\{0,1, \ldots, n-1\}$ the set $E_{k}^{(i)}$ is an open subset of $A(\Omega)$.

Proof Let $k \in \mathbb{N}$ and $i \in\{0,1, \ldots, n-1\}$. We will prove equivalently that $A(\Omega) \backslash E_{k}^{(i)}$ is a closed subset of $A(\Omega)$. Let $f_{m}$ be a sequence in $A(\Omega) \backslash E_{k}^{(i)}$ converging uniformly on $\bar{\Omega}$ to a function $f \in A(\Omega)$. For every $m \in \mathbb{N}$ there exist $\theta_{m} \in \mathbb{R}$ such that

$$
\left|\frac{u_{m}\left(\phi_{i}(y)\right)-u_{m}\left(\phi_{i}\left(\theta_{m}\right)\right)}{y-\theta_{m}}\right| \leq k,
$$

where $u_{m}=\operatorname{Re} f_{m}$. Since every $u_{m}$ is $2 \pi$ - periodic we may assume that every $\theta_{m} \in$ $[0,2 \pi]$ and thus the sequence $\left\{\theta_{m}\right\}$ has a convergent subsequence. Without loss of generality we assume that $\left\{\theta_{m}\right\}$ converges to a $\theta \in[0,2 \pi]$.

Let $y \in\left(\theta, \theta+\frac{1}{k}\right)$. We can easily deduce that there exists $m_{0} \in \mathbb{N}$, such that for every $m \geq m_{0}$ it is true that $y \in\left(\theta_{m}, \theta_{m}+\frac{1}{k}\right)$. Thus, applying (4.3) and using the convergence of $\left\{\theta_{m}\right\}$ to $\theta$ and the uniform convergence of $\left\{f_{m}\right\}$ to $f$ we have that

$$
\left|\frac{u\left(\phi_{i}(y)\right)-u\left(\phi_{i}(\theta)\right)}{y-\theta}\right| \leq k,
$$

where $u=\operatorname{Re} f$. Hence (4.4) holds for every $y \in\left(\theta, \theta+\frac{1}{k}\right)$ and thus $f \in A(\Omega) \backslash$ $E_{k}^{(i)}$. Therefore, $A(\Omega) \backslash E_{k}^{(i)}$ is a closed subset of $A(\Omega)$.

Theorem 4.3 The class

$$
S=\bigcap_{i=0}^{n-1} \bigcap_{k=1}^{\infty} E_{k}^{(i)}
$$

is a dense and $G_{\delta}$ subset of $A(\Omega)$.

Proof Lemma 4.2 indicates that for every $i \in\{0,1, \ldots, n-1\}$, the sets

$$
S_{i}=\bigcap_{k=1}^{\infty} E_{k}^{(i)}
$$

are $G_{\delta}$. We will prove that $S_{0}$ is dense in $A(\Omega)$.

Let $f \in A(\Omega)$ and $f_{i} \in A\left(\Omega_{i}\right)$ be such that $f=f_{0}+f_{1}+\ldots+f_{n-1}$. Such a decomposition is possible because $f$ is analytic on $\Omega, \hat{\mathbb{C}} \backslash \Omega$ has $n$ connected components, therefore we can apply the global Cauchy theorem on $\frac{f(\zeta)-f(z)}{\zeta-z}$, each time, integrating on appropriate curves that depend on $z \in \Omega$ [1]. Let also $g=$ 
$f_{1}+\ldots+f_{n-1}$. Since $\bar{\Omega} \subset \overline{\Omega_{0}}$ is compact, $\hat{\mathbb{C}} \backslash \Omega$ has $n$ connected components and $\phi_{0}^{-1}: \overline{\Omega_{0}} \rightarrow \mathbb{C}$ is a conformal map, the set $\phi_{0}^{-1}(\bar{\Omega}) \subset \bar{D} \subset \mathbb{C}$ is compact and $\hat{\mathbb{C}} \backslash \phi_{0}^{-1}(\bar{\Omega})$ has $n$ connected components. The function $g \circ \phi_{0}: \phi_{0}^{-1}(\bar{\Omega}) \rightarrow \mathbb{C}$ belongs to $A\left(\phi_{0}^{-1}(\Omega)\right)$ and by Mergelyan's theorem there exists a sequence $\left\{r_{m}\right\}$ of rationals functions with poles off $\phi_{0}^{-1}(\bar{\Omega})$ which converges to $g \circ \phi_{0}$ uniformly on $\phi_{0}^{-1}(\bar{\Omega})$. Thus, the sequence $\left\{r_{m} \circ \phi_{0}^{-1}\right\}$ converges to $g$ uniformly on $\bar{\Omega}$.

Since $f_{0} \in A\left(\Omega_{0}\right)$, by Thorem 3.1 there exists a sequence $\left\{g_{m}\right\}$, such that $g_{m} \in$ $A\left(\Omega_{0}\right)$ and $g_{m} \circ \phi_{0} \in Z(D)$, which converges to $f_{0}$. From the above we have that the sequence $\left\{g_{m}+r_{m} \circ \phi_{0}^{-1}\right\}$ converges uniformly to $f$ in $A(\Omega)$ and also that:

$$
\begin{aligned}
& \left|\frac{\operatorname{Re}\left(g_{m}+r_{m} \circ \phi_{0}^{-1}\right)\left(\phi_{0}(y)\right)-\operatorname{Re}\left(g_{m}+r_{m} \circ \phi_{0}^{-1}\right)\left(\phi_{0}(\theta)\right)}{y-\theta}\right| \\
& \geq\left|\frac{\operatorname{Re} g_{m}\left(\phi_{0}(y)\right)-\operatorname{Re} g_{m}\left(\phi_{0}(\theta)\right)}{y-\theta}\right| \\
& \quad-\left|\frac{\operatorname{Re}\left(r_{m} \circ \phi_{0}^{-1}\right)\left(\phi_{0}(y)\right)-\operatorname{Re}\left(r_{m} \circ \phi_{0}^{-1}\right)\left(\phi_{0}(\theta)\right)}{y-\theta}\right| \\
& =\left|\frac{\operatorname{Re} g_{m}\left(\phi_{0}(y)\right)-\operatorname{Re} g_{m}\left(\phi_{0}(\theta)\right)}{y-\theta}\right|-\left|\frac{\operatorname{Re} r_{m}(y)-\operatorname{Re} r_{m}(\theta)}{y-\theta}\right|
\end{aligned}
$$

for every $\theta, y \in \mathbb{R}, \theta \neq y$. From (4.7) and the fact that the functions $r_{m}$ are differentiable at the unit circle, every $g_{m}+r_{m} \circ \phi_{0}^{-1}$ belongs to $S_{0}$. Therefore, the set $S_{0}$ is dense.

Similarly, the sets $S_{1}, S_{2}, \ldots, S_{n-1}$ are dense and Baire's theorem completes the proof.

Proof of Theorem 4.1 It is easy to see that $S \cap i S$ is a subset of all functions $f \in A(\Omega)$ such that for every $i \in\{0,1, \ldots, n-1\}$ the functions $\left.\left(\operatorname{Re} f \circ \phi_{i}\right)\right|_{\mathbb{T}}$ and $\left(\operatorname{Im} f \circ \phi_{i}\right) \Gamma_{\mathbb{T}}$ have no derivatives at any point. Since $S$ is $G_{\delta}$ and dense in $A(\Omega)$ and multiplication by $i$ is an automorphism of $A(\Omega)$, we conclude that $i S$ is $G_{\delta}$ and dense in $A(\Omega)$. Applying Baire's category theorem we have that $S \cap i S$ is $G_{\delta}$ dense in $A(\Omega)$.

It is natural to ask if our results can be generalised to domains bounded by an infinite number of Jordan curves.

Question 4.4 Can our results be generalised to domains $\Omega$ bounded by an infinite number of Jordan curves?

\section{The case of several variables}

Let $I$ be a non empty finite or countably infinite set and $\left\{\Omega_{i}\right\}_{i \in I}$ be a family of domains in $\mathbb{C}$, all of them bounded by a finite number of disjoint Jordan curves as in Sect. 4. We denote by $k_{i}$ the degree of connectivity of $\Omega_{i}$. In accordance to the notation of the previous sections, we denote by $V_{i, j}, i \in I, j \in\left\{0, \ldots, k_{i}-1\right\}$ the connected 
components of $\hat{\mathbb{C}} \backslash \overline{\Omega_{i}}$ and $\Omega_{i, j}=\hat{\mathbb{C}} \backslash \overline{V_{i, j}}$. Let also $\infty \in \Omega_{i, 0}$ and $\phi_{i, j}: \bar{D} \rightarrow \overline{\Omega_{i, j}}$ be some fixed Riemann maps.

We consider the spaces $\Omega=\prod_{i \in I} \Omega_{i}, \bar{\Omega}=\prod_{i \in I} \overline{\Omega_{i}}$ and $b_{0} \Omega=\prod_{i \in I} \partial \Omega_{i}$, the distinguished boundary of $\Omega$, all of them endowed with the product topology. According to $[4,5]$ and $[8]$ we give the following definition.

Definition 5.1 A function $f: \bar{\Omega} \rightarrow \mathbb{C}$ belongs to the space $A(\Omega)$ if it is continuous on $\bar{\Omega}$, endowed with the product topology, and separately holomorphic on $\Omega$. By separately holomorphic we mean that if all coordinates but one are fixed, say the coordinate $i_{0}$, then the restriction of $f$ is a holomorphic function of the variable $z_{i_{0}}$ in $\Omega_{i_{0}}$. We endow the space $A(\Omega)$ with the supremum norm.

We also consider the set

$$
R=\prod_{i \in I}\left\{0, \ldots, k_{i}-1\right\}
$$

and the functions

$$
\begin{gathered}
\phi_{r}: \bar{D}^{I} \longrightarrow \overline{\Omega_{1, r_{1}}} \times \overline{\Omega_{2, r_{2}}} \times \cdots=\prod_{i \in I} \bar{\Omega}_{i, r_{i}} \\
\left(z_{i}\right)_{i \in I} \longmapsto\left(\phi_{i, r_{i}}\left(z_{i}\right)\right)_{i \in I}
\end{gathered}
$$

where $r=\left(r_{i}\right)_{i \in I} \in R$.

We are going to prove that, generically, for every $f \in A(\Omega)$ the functions $\operatorname{Re}(f \circ$ $\left.\phi_{r}\right) \uparrow_{\mathbb{T}^{I}}$ and $\operatorname{Re}\left(f \circ \phi_{r}\right) \uparrow_{\mathbb{T}^{I}}$ have no directional derivatives for any $r \in R$ and any direction $v \in \ell^{\infty}(I) \backslash\{0\}$. In order to do so, we will first treat the case where $I$ is a finite set and separately the case where $I$ is a countably infinite set.

\subsection{Finitely many variables}

Let $I$ be a non empty finite set.

We consider the sets

$$
\begin{aligned}
D_{n, k, s}(I) & =\left\{u \in C_{\mathbb{R}}\left(\mathbb{T}^{I}\right): \text { for every } \theta \in \mathbb{R}^{I}\right. \text { and every direction } \\
v & \in \mathbb{R}^{I} \text { with } \frac{1}{s} \leq\left|v_{k}\right| \leq s \text { and }\left|v_{j}\right| \leq s \text { for every } j \in I \text { there exists } \\
y & \left.\in\left(\theta-\frac{1}{n} v, \theta+\frac{1}{n} v\right) \text { such that }|u(\theta)-u(y)|>n\|y-\theta\|_{\infty}\right\}
\end{aligned}
$$

for some $n, s \in \mathbb{N}$ and $k \in I$. Also consider:

$$
E_{n, k, s}^{(r)}(I)=\left\{f \in A(\Omega): \operatorname{Re}\left(f \circ \phi_{r}\right) \uparrow_{\mathbb{T}^{I}} \in D_{n, k, s}\right\}
$$

for some $r \in R$. 
Lemma 5.2 For every $n, s \in \mathbb{N}, k \in I$ and $r \in R$ the set $E_{n, k, s}^{(r)}(I)$ is an open subset of $A(\Omega)$.

Proof We will prove that the set $A(\Omega) \backslash E_{n, k, s}^{(r)}(I)$ is closed. We consider a sequence $\left\{f_{m}\right\}$ such that every $f_{m} \in A(\Omega) \backslash E_{n, k, s}^{(r)}(I)$, which uniformly converges to a function $f \in A(\Omega)$. For each $m$ there exists $\theta^{(m)} \in \mathbb{T}^{I}$ and a direction $v^{(m)} \in \mathbb{R}^{I}$ with $\left|v_{j}^{(m)}\right| \leq s$ for all $j \in I$ and $\frac{1}{s} \leq\left|v_{k}^{(m)}\right| \leq s$, such that:

$$
\left|\left(u_{m} \circ \phi_{r}\right)(y)-\left(u_{m} \circ \phi_{r}\right)\left(\theta^{(m)}\right)\right| \leq n\left\|y-\theta^{(m)}\right\|_{\infty}
$$

for every $y \in\left(\theta^{(m)}-\frac{1}{n} v^{(m)}, \theta^{(m)}+\frac{1}{n} v^{(m)}\right)$, where $u_{m}=\operatorname{Re} f_{m}$. But $\mathbb{T}^{I}$ and the set $\left\{v \in \mathbb{R}^{I}:\left|v_{j}\right| \leq s, \forall j \in I\right.$ and $\left.\frac{1}{s} \leq\left|v_{k}\right| \leq s\right\}$ are compact. Thus, the sequences $\left\{\theta^{(m)}\right\}_{m \in \mathbb{N}}$ and $\left\{v^{(m)}\right\}_{m \in \mathbb{N}}$ have converging subsequences. Without loss of generality we assume that $\theta^{(m)} \rightarrow \theta \in \mathbb{T}^{I}$ and $v^{(m)} \rightarrow v \in \mathbb{R}^{I}$ with $\left|v_{j}\right| \leq s$ for all $j \in I$ and $\frac{1}{s} \leq\left|v_{k}\right| \leq s$.

If $y \in\left(\theta-\frac{1}{n} v, \theta+\frac{1}{n} v\right)$, then there exists $-\frac{1}{n}<t<\frac{1}{n}$ such that

$$
y=\theta+t v=\lim _{m \rightarrow \infty}\left(\theta^{(m)}+t v^{(m)}\right) .
$$

Since $f_{m}$ converges uniformly to $f$, it follows that $u_{m}$ converge uniformly to $u$, where $u=\operatorname{Re} f$ and $\phi_{r}$ is continuous which implies that $\phi_{r}\left(\theta^{(m)}+t v^{(m)}\right) \rightarrow \phi_{r}(\theta+$ $t v)$, as $m \rightarrow \infty$. The above combined with (5.5) imply that

$$
\left|\left(u \circ \phi_{r}\right)(y)-\left(u \circ \phi_{r}\right)(\theta)\right| \leq n\|y-\theta\|_{\infty}
$$

and therefore $f \in A(\Omega) \backslash E_{n, k, s}^{(r)}(I)$ which proves the lemma.

Now let

$$
S_{k, s}^{(r)}(I)=\bigcap_{n=1}^{\infty} E_{n, k, s}^{(r)}(I)
$$

for all $r \in R, k \in I$ and $s \in \mathbb{N}$. Obviously, $S_{k, s}^{(r)}(I)$ is $G_{\delta}$ in $A(\Omega)$.

Lemma 5.3 For all $r \in R$ and $k, s \in I$ the class $S_{k, s}^{(r)}(I)$ is dense in $A(\Omega)$.

Proof Let us show that $S_{k, s}^{(r)}(I)$ is non empty. We have proved in a previous section that there is a function $g \in A\left(\Omega_{k}\right)$ such that

$$
\limsup _{y \rightarrow \theta^{+}}\left|\frac{\operatorname{Re}\left(g \circ \phi_{k, r_{k}}\right)(y)-\operatorname{Re}\left(g \circ \phi_{k, r_{k}}\right)(\theta)}{y-\theta}\right|=+\infty
$$


for all $\theta \in \mathbb{R}$. The above implies that for all $\theta \in \mathbb{R}$ :

$$
\limsup _{t \rightarrow 0^{+}}\left|\frac{\operatorname{Re}\left(g \circ \phi_{k, r_{k}}\right)(\theta+t \alpha)-\operatorname{Re}\left(g \circ \phi_{k, r_{k}}\right)(\theta)}{t \alpha}\right|=+\infty
$$

for any $\alpha \in \mathbb{R}$ with $\frac{1}{s} \leq|\alpha| \leq s$.

Now consider the function $f: \bar{\Omega} \longrightarrow \mathbb{C}$ which maps $\left(z_{i}\right)_{i \in I}$ to $g\left(z_{k}\right)$. We will prove that $f \in S_{k, s}^{(r)}(I)$. Let $\theta \in \mathbb{R}^{I}$ and $v \in \mathbb{R}^{I}$ such that $\left|v_{j}\right| \leq s$ for all $j \in I$ and $\frac{1}{s} \leq\left|v_{k}\right| \leq s$. We observe that

$$
\left|\frac{u_{r}(\theta+t v)-u_{r}(\theta)}{t\|v\|_{\infty}}\right|=\frac{\left|v_{k}\right|}{\|v\|_{\infty}}\left|\frac{\tilde{u}_{k, r_{k}}\left(\theta_{k}+t v_{k}\right)-\tilde{u}_{k, r_{k}}\left(\theta_{k}\right)}{t v_{k}}\right|,
$$

does not remain bounded as $t \rightarrow 0$, where $u_{r}=\operatorname{Re} f \circ \phi_{r}$ and $\tilde{u}_{k, r_{k}}=\operatorname{Re} f \circ \phi_{k, r_{k}}$. Thus $f \in S_{k, s}^{(r)}(I)$ and $S_{k, s}^{(r)}(I) \neq \emptyset$.

Let us now show that $S_{k, s}^{(r)}(I)$ is dense in $A(\Omega)$. We remind the reader that

$$
\begin{gathered}
\phi_{r}: \bar{D}^{I} \longrightarrow \overline{\Omega_{1, r_{1}}} \times \overline{\Omega_{2, r_{2}}} \times \cdots=\prod_{i \in I} \bar{\Omega}_{i, r_{i}} \\
\left(z_{i}\right)_{i \in I} \longmapsto\left(\phi_{i, r_{i}}\left(z_{i}\right)\right)_{i \in I}
\end{gathered}
$$

and that the function

$$
\begin{aligned}
& \phi_{r}^{-1}: \overline{\Omega_{1, r_{1}}} \times \overline{\Omega_{2, r_{2}}} \times \cdots \longrightarrow \bar{D}^{I} \\
& \left(w_{i}\right)_{i \in I} \longmapsto\left(\phi_{i, r_{i}}^{-1}\left(w_{i}\right)\right)_{i \in I}
\end{aligned}
$$

is the inverse of $\phi_{r}$. It easily follows that $\phi_{r} \in A\left(D^{I}\right)$ and $\phi_{r}^{-1} \in A\left(\Omega_{1, r_{1}} \times \Omega_{2, r_{2}} \times \cdots\right)$. Now, note that $\phi_{r}^{-1}(\bar{\Omega})=\prod_{i \in I} \phi_{i, r_{i}}^{-1}\left(\overline{\Omega_{i}}\right)$ where $\phi_{i, r_{i}}^{-1}\left(\overline{\Omega_{i}}\right)$ is a compact set in $\mathbb{C}$ of finite connectivity and consider the functions

$$
\begin{aligned}
& \widetilde{\phi}_{r}: \phi_{r}^{-1}(\bar{\Omega}) \longrightarrow \bar{\Omega} \\
& z \longmapsto \phi_{r}(z)
\end{aligned}
$$

and

$$
\begin{aligned}
& \widetilde{\phi_{r}^{-1}}: \bar{\Omega} \longrightarrow \phi_{r}^{-1}(\bar{\Omega}) \\
& z \longmapsto \phi_{r}^{-1}(z)
\end{aligned}
$$

We observe that $\widetilde{\phi_{r}^{-1}}$ is the inverse of $\widetilde{\phi}_{r}$. Also, note that if $h \in A(\Omega)$, then $h \circ \widetilde{\phi}_{r} \in$ $A\left(\phi_{r}^{-1}(\bar{\Omega})\right)$. Since $\phi_{r}^{-1}(\bar{\Omega})$ is a product of domains in $\mathbb{C}$ bounded by a finite number 
of disjoint Jordan curves, then according to the result of [4] in the case of finitely many variables, the set $U$ of functions which are finite sums of finite products of rational functions of one complex variable $z_{i}$ with poles off $\Omega_{i}$ is dense in $A\left(\phi_{r}^{-1}(\bar{\Omega})\right)$. Hence, the set $f+U \circ \widetilde{\phi_{r}^{-1}}$ is dense in $A(\Omega)$. If $q \in U$, then for every $t \in \mathbb{R}, \theta \in \mathbb{R}^{I}$ and $v \in \mathbb{R}^{I}$ with $\left|v_{j}\right| \leq s$ for all $j \in I$ and $\frac{1}{s} \leq\left|v_{k}\right| \leq s$ we have that:

$$
\left|\frac{\left(q \circ \widetilde{\phi_{r}^{-1}}\right)\left(\phi_{r}(\theta+t v)\right)-\left(q \circ \widetilde{\phi_{r}^{-1}}\right)\left(\phi_{r}(\theta)\right)}{t\|v\|_{\infty}}\right|=\frac{1}{\|v\|_{\infty}}\left|\frac{q(\theta+t v)-q(\theta)}{t}\right|
$$

remains bounded. This follows from the fact that every rational function of one complex variable $z_{i}$ which appears in $q$ has poles off $\overline{\Omega_{i}}$. Thus, the function $p(t)=$ $q(\theta+t v)$ is differentiable at 0 . Therefore, $f+U \circ \widetilde{\phi_{r}^{-1}} \subset S_{k, s}^{(r)}(I)$, which completes the proof.

Baire's theorem combined with Lemmas 5.2 and 5.3 imply the following theorem.

Theorem 5.4 The class

$$
S(I)=\bigcap_{r \in R} \bigcap_{k \in I} S_{s \in \mathbb{N}}^{(r)}(I)
$$

is a dense and $G_{\delta}$ subset of $A(\Omega)$.

Consequently, $S(I) \cap i S(I)$ is dense and $G_{\delta}$ in $A(\Omega)$ and it is a subset of the set of all functions $f \in A(\Omega)$ such that both $\operatorname{Re}\left(f \circ \phi_{r}\right) \uparrow_{\mathbb{T}^{I}}$ and $\operatorname{Im}\left(f \circ \phi_{r}\right) \uparrow_{\mathbb{T}^{I}}$ are nowhere differentiable for any $r \in R$ and any direction $v \in \ell^{\infty}(I) \backslash\{0\}$.

It is natural to ask if our results hold for domains bounded by a countably infinite number of disjoint Jordan curves.

\subsection{Infinitely many variables}

Let $I$ be an countably infinite set.

Our goal is to generalize the result of the previous subsection to the case of infinite many variables, namely we intend to prove that the set of functions $f \in A(\Omega)$ such that for every $\theta \in \prod_{i \in I} \partial \Omega_{i}$ and direction $u \in \ell^{\infty} \backslash\{0\}$,

$$
\limsup _{t \rightarrow 0} \frac{|\operatorname{Re}(f)(\theta+t v)-\operatorname{Re}(f)(\theta)|}{\|t v\|_{\infty}}=+\infty
$$

and

$$
\limsup _{t \rightarrow 0} \frac{|\operatorname{Im}(f)(\theta+t v)-\operatorname{Im}(f)(\theta)|}{\|t v\|_{\infty}}=+\infty
$$


is dense and $G_{\delta}$. For technical reasons we need to replace the $\ell^{\infty}$-norm in the above definition by the metric

$$
\rho\left(\left(z_{i}\right)_{i \in \mathbb{N}},\left(w_{i}\right)_{i \in \mathbb{N}}\right)=\sum_{i=1}^{\infty} \frac{\left|z_{i}-w_{i}\right|}{2^{i}}
$$

which defines the pointwise convergence. However, the two aforementioned sets coincide, since the $\ell^{\infty}$-norm and $\rho$ are equivalent in every line of the form $\theta+\mathbb{R} v$. Hence, it suffices to prove that the latter set is dense and $G_{\delta}$.

Let us start by defining the sets

$$
\begin{aligned}
D_{n, k, s}(I) & =\left\{u \in C_{\mathbb{R}}\left(\mathbb{T}^{I}\right): \text { for every } \theta \in \mathbb{R}^{I}\right. \text { and every direction } \\
v & \in \mathbb{R}^{I} \text { with }\left|v_{j}\right| \leq s \text { for any } j \in I \text { and } \frac{1}{s} \leq\left|v_{k}\right| \leq s \text { there exists } \\
y & \left.\in\left(\theta-\frac{1}{n} v, \theta+\frac{1}{n} v\right) \text { such that }|u(\theta)-u(y)|>n \rho(y, \theta)\right\}
\end{aligned}
$$

and

$$
E_{n, k, s}(I)=\bigcap_{r \in R}\left\{f \in A(\Omega): \operatorname{Re}\left(f \circ \phi_{r}\right) \uparrow_{\mathbb{T}^{I}} \in D_{n, k, s}\right\},
$$

for $n, s \in \mathbb{N}$ and $k \in I$.

Lemma 5.5 For every $n, s \in \mathbb{N}$ and $k \in I$ the set $E_{n, k, s}(I)$ is an open subset of $A(\Omega)$.

Proof We will prove that the set $A(\Omega) \backslash E_{n, k, s}(I)$ is closed. We consider a sequence $\left\{f_{m}\right\}$ such that every $f_{m} \in A(\Omega) \backslash E_{n, k, s}(I)$, which converges to a function $f \in A(\Omega)$. For each $m$ there exist $r^{(m)} \in R, \theta^{(m)} \in \mathbb{T}^{\mathbb{N}}$ and a direction $v^{(m)} \in \mathbb{R}^{\mathbb{N}}$ with $\left|v_{j}^{(m)}\right| \leq s$ for all $j \in I$ and $\frac{1}{s} \leq\left|v_{k}^{(m)}\right| \leq s$, such that

$$
\left|\left(u_{m} \circ \phi_{r^{(m)}}\right)(y)-\left(u_{m} \circ \phi_{r^{(m)}}\right)\left(\theta^{(m)}\right)\right| \leq n \rho\left(y, \theta^{(m)}\right)
$$

for every $y \in\left(\theta^{(m)}-\frac{1}{n} v^{(m)}, \theta^{(m)}+\frac{1}{n} v^{(m)}\right)$, where $u_{m}=\operatorname{Re} f_{m}$. But $R, \mathbb{T}^{\mathbb{N}}$ and the set $\left\{v \in \mathbb{R}^{\mathbb{N}}:\left|v_{j}\right| \leq s, \forall j \in I\right.$ and $\left.\frac{1}{s} \leq\left|v_{k}\right| \leq s\right\}$ are metrizable and compact by Tychonoff's theorem. Thus, they have converging subsequences. Without loss of generality we assume that $r^{(m)} \rightarrow r \in R, \theta^{(m)} \rightarrow \theta \in \mathbb{R}^{\mathbb{N}}$ and $v^{(m)} \rightarrow v$, where $v \in \mathbb{R}^{\mathbb{N}}$ and $\left|v_{j}\right| \leq s$ for all $j \in I$ and $\frac{1}{s} \leq\left|v_{k}\right| \leq s$.

$$
\begin{gathered}
\text { If } y \in\left(\theta-\frac{1}{n} v, \theta+\frac{1}{n} v\right) \text {, then there exists a } t \in \mathbb{R} \text { with }-\frac{1}{n}<t<\frac{1}{n} \text { such that: } \\
y=\theta+t v=\lim _{m \rightarrow \infty}\left(\theta^{(m)}+t v^{(m)}\right) \text {. }
\end{gathered}
$$


Let $i \in I$. Since $R=\prod_{i \in I}\left\{0, \ldots, k_{i}-1\right\}$ is a product of finite sets and $r^{(m)} \rightarrow r \in \mathbb{R}$, there exists $m_{0} \in \mathbb{N}$, such that

$$
\phi_{i, r_{i}(m)}=\phi_{i, r_{i}}, \forall m \geq m_{0} .
$$

Since $\phi_{i, r_{i}}$ is continuous, we have that

$$
\phi_{i, r_{i}(m)}\left(\theta_{i}^{(m)}+t v_{i}^{(m)}\right) \rightarrow \phi_{i, r_{i}}\left(y_{i}\right), \quad \phi_{i, r_{i}}^{(m)}\left(\theta_{i}^{(m)}\right) \rightarrow \phi_{i, r_{i}}\left(\theta_{i}\right)
$$

Hence,

$$
\phi_{r_{(m)}}\left(y^{(m)}\right) \rightarrow \phi_{r}(y), \quad \phi_{r_{(m)}}\left(\theta^{(m)}\right) \rightarrow \phi_{r}(\theta)
$$

in the product topology. Therefore, letting $m \rightarrow \infty$ we see that the left hand side of (5.20) converges to $\left|\left(u \circ \phi_{r}\right)(y)-\left(u \circ \phi_{r}\right)(\theta)\right|$ where $u=\operatorname{Re} f$, since $f_{m} \rightarrow f$ uniformly, while the right hand side converges to $\rho(y, \theta)$. Hence,

$$
\left|\left(u \circ \phi_{r}\right)(y)-\left(u \circ \phi_{r}\right)(\theta)\right| \leq n \rho(y, \theta),
$$

which implies that $f \in A(\Omega) \backslash E_{n, k, s}(I)$ and that the set $A(\Omega) \backslash E_{n, k, s}(I)$ is closed.

Theorem 5.6 The class

$$
S(I)=\bigcap_{k \in I} \bigcap_{s=1}^{\infty} \bigcap_{n=1}^{\infty} E_{n, k, s}(I)
$$

is a dense and $G_{\delta}$ subset of $A(\Omega)$.

Proof Lemma 5.5 implies that $S(I)$ is a $G_{\delta}$. Thus, we only have to prove that $S(I)$ is dense. Let $F \in A(\Omega)$. From [4] there exist a sequence of finite sets $\left\{I_{n}\right\}, I_{n} \subset I$, and a sequence $\left\{F_{n}\right\}$ in $A(\Omega)$, such that each $F_{n}$ depends only on the variables $z_{i}$, $i \in I_{n}$ and $F_{n} \rightarrow F$ uniformly. Let $f_{n}$ be the restriction of $F_{n}$ to $\prod_{i \in I_{n}} \Omega_{i}$. From Theorem 5.4 there exists a sequence $g_{n} \in S\left(I_{n}\right)=\bigcap_{r \in R\left(I_{n}\right)} \bigcap_{k \in I_{n}} \bigcap_{s=1}^{\infty} S_{k, s}^{(r)}\left(I_{n}\right)$, where $R\left(I_{n}\right)=\prod_{i \in I_{n}}\left\{0, \ldots, k_{i}-1\right\}$, such that $\left\|f_{n}-g_{n}\right\|_{\infty} \rightarrow 0$. It is easy to see that the functions $G_{n}: \bar{\Omega} \rightarrow \mathbb{C}$ which map $\left(z_{i}\right)_{i \in I}$ to $g_{n}\left(\left(z_{i}\right)_{i \in I_{n}}\right)$ respectively belong to $S(I)=\bigcap_{k \in I} \bigcap_{s=1}^{\infty} \bigcap_{n=1}^{\infty} E_{n}(I)$ and $G_{n} \rightarrow F$. The proof is complete.

The analogue of Theorem 4.1 follows immediately: if $I$ is a non empty finite set or an infinitely countable set, then the set

$$
T(I)=S(I) \cap i S(I)
$$

is a dense and $G_{\delta}$ subset of $A(\Omega)$. This gives the non directional differentiability at every boundary point of both real and imaginary part of the function in every direction in $\ell^{\infty}(I) \backslash\{0\}$. 
Remark 5.7 In [3] it is claimed that the set of functions $f \in A\left(D^{\mathbb{N}}\right)$ for which for every $\theta \in \mathbb{R}^{\mathbb{N}}$ and every direction $v \in \mathbb{R}^{\mathbb{N}}$ with $\|v\|_{\infty}=1$ and $\frac{1}{2} \leq\left|v_{k}\right| \leq 1$ for a fixed $k \in \mathbb{N}$ there exists $y \in\left(\theta-\frac{1}{n} v, \theta+\frac{1}{n} v\right)$ such that

$$
|u(\theta)-u(y)|>n\|y-\theta\|_{\infty}
$$

is open in $A\left(D^{\mathbb{N}}\right)$. The authors' proof uses the wrong statement that the set $\{v \in$ $\mathbb{R}^{\mathbb{N}}$ with $\|v\|_{\infty}=1$ and $\left.\frac{1}{2} \leq\left|v_{k}\right| \leq 1\right\}$ for a fixed $k \in \mathbb{N}$ is compact in $\mathbb{R}^{\mathbb{N}}$ endowed with the product topology. However, their proof works just fine for every compact set of directions with non zero $k$-coordinate. According to our Theorem 5.6 their statement is correct.

Acknowledgements We would like to express our gratidute to Professor Vassili Nestoridis for introducing us to the problem as well as for his guidance throughout the creation of this paper. We would also like to thank A. Eskenazis for his suggestions.

Open Access This article is distributed under the terms of the Creative Commons Attribution 4.0 International License (http://creativecommons.org/licenses/by/4.0/), which permits unrestricted use, distribution, and reproduction in any medium, provided you give appropriate credit to the original author(s) and the source, provide a link to the Creative Commons license, and indicate if changes were made.

\section{References}

1. Ahlfors, L.V.: Complex Analysis, 3rd edn. Mc Graw-Hill Book Co, New York (1978)

2. Banach, S.: Über die Baire'sche Kategorie gewisser Funktionenmengen. Stud. Math. 3, 174-179 (1931)

3. Eskenazis, A., Makridis, K.: Topological genericity of nowhere differentiable functions in the disc and polydisc algebras. JMAA 420(1), 435-446 (2014)

4. Falcó, J., Nestoridis, V.: Rational approximation on products of planar domains. Oberwolfach Prepr. 5, 1-9 (2016)

5. Gauthier, P.M., Nestoridis, V.: Density of polynomials in classes of functions on products of planar domains. JMAA 433(1), 282-290 (2016)

6. Hardy, G.H.: Weierstrass's non-differentiable function. Trans. Am. Math. Soc. 17, 301-325 (1916)

7. Koosis, P.: Introduction to $H_{p}$ Spaces. Cambridge University Press, Cambridge (1998)

8. Makridis, K., Nestoridis, V.: Sets of uniqueness for uniform limits of polynomials in several complex variables. JMAA 432(2), 994-1004 (2015)

9. Mazurkiewicz, S.: Sur les fonctions non dérivables. Stud. Math. 3, 92-94 (1931) 